\title{
Communications Problem Identified
}

National Cancer Institute

\section{Source}

National Cancer Institute. Communications Problem Identified. NCI Thesaurus. Code C92034.

Devices that do not send or receive adequate signals (this speaks to the interoperability between devices). 